\title{
Mindfulness e inteligencia emocional en la prevención de recaídas en personas en tratamiento: una revisión*
}

\author{
Julia Lizeth Villarrea Mata ${ }^{1}$ \\ (D) https://orcid.org/0000-0002-8829-0537 \\ Edna Idalia Paulina Navarro Oliva² \\ Di https://orcid.org/0000-0002-2695-8220 \\ Edgar Bresó Esteve ${ }^{3}$ \\ (D) https://orcid.org/0000-0003-1332-7689 \\ Linda Azucena Rodríguez Puente ${ }^{2}$ \\ (D) https://orcid.org/0000-0003-4040-6171 \\ María Magdalena Alonso-Castillo ${ }^{1}$ \\ (D) https://orcid.org/0000-0002-7197-8116 \\ Reyna Torres Obregón ${ }^{2}$ \\ (D) https://orcid.org/0000-0003-3546-6970
}

\begin{abstract}
Objetivo: analizar la evidencia empírica disponible sobre los programas de intervención de Mindfulness e Inteligencia Emocional para la prevención de recaídas en personas en tratamiento. Metodología: en la estrategia de búsqueda se incluyeron descriptores en los idiomas inglés (Mindfulness, emotional intelligence, relapse prevention) y español (atención plena, inteligencia emocional, prevención de la recaída). Resultados: se identifió efecto significativo de la práctica de Mindfulness, sobre la inteligencia eemocional al apoyar en la regulación de las emociones y su vez prevenir posibles recaídas. Conclusion: continuar investigando sobre el Mindfulness y sus efectos sobre la inteligencia emocional en diferentes contextos.
\end{abstract}

Descriptores: Mindfulness; Emotional intelligence; Relapse prevention

\section{Como citar este artigo}

Villarreal-Mata JL, Navarro-Oliva EIP, Bresó EE, Rodríguez LA, Alonso-Castillo MM, Torres OR. Mindfulness and emotional intelligence in the prevention of relapses in people in treatment: a review. SMAD, Rev Eletrônica Saúde Mental Álcool Drog. 2020;16(3):81-87. doi: https://dx.doi.org/10.11606/issn.1806-6976.smad.2020.167320 


\title{
Mindfulness and emotional intelligence in the prevention of relapses in people in treatment: a review
}

\begin{abstract}
Objective: to analyze the empirical evidence available on Mindfulness and Emotional Intelligence intervention programs for relapse prevention in people in treatment. Methodology: descriptors in English (Mindfulness, emotional intelligence, relapse prevention) and Spanish (mindfulness, emotional intelligence, relapse prevention) languages were included in the search strategy. Results: a significant effect of Mindfulness practice was identified on emotional intelligence by supporting the regulation of emotions and preventing possible relapses. Conclusion: continue researching Mindfulness and its effects on emotional intelligence in different contexts.
\end{abstract}

Descriptores: Mindfulness; Emotional Intelligence; Relapse Prevention.

\section{Mindfulness e inteligência emocional na prevenção de recaídas em pessoas em tratamento: uma revisão}

\footnotetext{
Objetivo: analisar as evidências empíricas disponíveis nos programas de intervenção Mindfulness e Emotional Intelligence para prevenção de recaídas em pessoas em tratamento. Metodologia: os descritores nos idiomas inglês (atenção plena, inteligência emocional, prevenção de recaídas) e espanhol (atenção plena, inteligência emocional, prevenção de recaídas) foram incluídos na estratégia de busca. Resultados: um efeito significativo da prática da atenção plena foi identificado na inteligência emocional, apoiando a regulação das emoções e prevenindo possíveis recaídas. Conclusão: continuar pesquisando Mindfulness e seus efeitos na inteligência emocional em diferentes contextos.
}

Descriptores: Atenção Plena; Inteligência Emocional; Prevenção de Recaídas. 


\section{Introducción}

La dependencia al alcohol y a las drogas ilícitas son graves problemas de salud pública en todos los países del mundo debido a las consecuencias generadas a la salud física y mental de los consumidores y de quiénes los rodean. De acuerdo con la Organización Mundial de la Salud, aproximadamente tres millones de personas mueren en el mundo cada año a causa del consumo de alcohol, lo que representa el $5.1 \%$ de la carga de morbilidad. Así mismo, el $1.5 \%$ de las defunciones totales son atribuibles al consumo de drogas ilícitas(1)

A nivel mundial, aproximadamente 275 millones de personas, es decir, alrededor del $5.6 \%$ de la población mundial de entre 15 y 64 años ha consumido alguna droga por lo menos una vez en la vida. Es importante resaltar, que, de estos consumidores, aproximadamente 31 millones padecen trastornos provocados por el consumo, que podrían requerir tratamiento para reducir el consumo o prevenir recaídas(2).

En México, el $71 \%$ de la población adulta de entre 18 y 65 años ha consumido alcohol alguna vez en la vida, el $49.1 \%$ en el último año y el $35.9 \%$ en el último mes. Así mismo, la ingesta excesiva de alcohol en este grupo de edad incrementó un 8.2\% del año 2011 al 2016. Es importante mencionar que, en esta población consumidora de alcohol en el último año, cerca del 3\% ha solicitado tratamiento, mientras que de las personas que ya presentan dependencia al alcohol aumenta a un $13.9 \%$ y se destaca que las mujeres son quienes menos lo solicitan $(8.1 \%)^{(3)}$.

En relación con el consumo de drogas ilícitas, el $10.3 \%$ de la población han consumido alguna vez en la vida, el $2.9 \%$ en el último año y el $1.5 \%$ en el último mes, es importante destacar que el $0.6 \%$ ya presenta una dependencia al consumo de drogas ilícitas, de los cuales solo el $20.3 \%$ han solicitado tratamiento en el último año; de los usuarios con dependencia que estuvieron en tratamiento, el $32.4 \%$ estuvieron en un anexo, el $24.8 \%$ en tratamiento por desintoxicación y el $13.2 \%$ llevaron tratamiento psiquiátrico o residencial(3).

El tratamiento para la dependencia a las drogas tiene como finalidad proporcionar a la persona estrategias, herramientas o competencias que ayuden al manejo del consumo compulsivo de la droga y en la reducción de la búsqueda; dicho tratamiento puede llevarse a cabo en una gran variedad de entornos, de diversas formas y por diferentes periodos de tiempo ${ }^{(4)}$. Sin embargo, el consumo dependiente de drogas suele considerarse como un trastorno crónico caracterizado por constantes recaídas y por lo regular, no basta con un ciclo de tratamiento(4).

La evidencia menciona que la dependencia a las drogas parte de la necesidad de escapar de las emociones consideradas como negativas, que dañan o alteran la salud mental de las personas, se ha observado que la no regulación de las emociones es una de las principales causas asociadas al consumo, ya que las personas consumidoras al experimentar dificultades 0 situaciones consideradas como estresantes, dolorosas o angustiantes buscan atenuar o minimizar dichas emociones y al no contar con habilidades que permitan la regulación emocional pueden llegar a utilizar estrategias desadaptativas como el abuso de drogas o el presentar alguna recaída en el consumo(5-14).

Por lo anterior es importante utilizar herramientas que regulen o gestionen las emociones, es decir que aumenten la Inteligencia Emocional (IE) de las personas consumidoras. La IE es definida como un conjunto de conocimientos y habilidades, referente al ámbito emocional y social que influyen en la capacidad general para afrontar efectivamente las demandas del contexto; dicha habilidad se basa en la capacidad del individuo de ser consciente, comprender, controlar, regular y expresar sus emociones de manera efectiva(15).

Se menciona que la IE esta compuesta por cinco elementos: en primer lugar, el componente intrapersonal, que se refiere a la comprensión de sí mismo, asertividad, auto concepto, autorrealización e independencia, es decir la habilidad de ser consciente, de comprender y relacionarse con otros. El segundo componente es el interpersonal, que al incluir la empatía, relaciones interpersonales y responsabilidad social implica la habilidad para regular emociones fuertes y controlar los impulsos ${ }^{(15)}$.

El tercer componente hace referencia a adaptabilidad o ajuste que hace referencia a la solución de problemas, es decir la capacidad para identificar y definir los problemas y generar e implementar soluciones efectivas, incluyendo además la flexibilidad para ajustar o regular las emociones, así como para evaluar lo que se experimenta y lo que en realidad existe. El cuarto componente corresponde al estado de ánimo, que está constituido por la habilidad para adaptarse a los cambios y resolver problemas de naturaleza personal y social; y finalmente el componente del manejo de estrés, que involucra la habilidad de tener una visión positiva y optimista(15).

Una de las herramientas que ha sido utilizada en los últimos años a nivel mundial es la práctica de Mindfulness, el cual ha sido integrado a algunos programas de intervención para la prevención de recaídas en personas consumidoras de alcohol y drogas ilícitas mostrando resultados significativos en la regulación de las emociones, que a su vez impactan indirectamente en la prevención de la recaída. El estado de Mindfulness considera dos elementos básicos en el proceso de regulación emocional, la autorregulación de la atención en el momento presente 
y la minimización de los juicios de valor ${ }^{(16)}$; en este sentido la práctica de Mindfulness conecta a la persona con sus emociones de forma compasiva y no reactiva, en donde le permite desvincularse de los pensamientos y procesos emociones ${ }^{(17)}$.

Por lo anterior, el objetivo de la presente revisión es analizar la evidencia empírica disponible sobre los programas de intervención basados en Mindfulness e Inteligencia Emocional para la prevención de recaídas en personas en tratamiento.

\section{Método}

La revisión de literatura se realizó de acuerdo con los pasos propuestos por el Manual Cochrane ${ }^{(18)}$. Primeramente, se formuló la pregunta de investigación con la finalidad de dar respuesta a los objetivos planteados, seguido a esto, se definieron los criterios de selección, se realizó la búsqueda y selección de artículos, así como la evaluación. Posteriormente, se llevó a cabo el análisis de datos obtenidos y finalmente, se describió la síntesis y se presentan los resultados(18-19).

Los criterios de inclusión para la selección de artículos fueron los siguientes: delimitados a los últimos cinco años de publicación (2015 al 2020), artículos en idioma inglés y español que fueron realizados por profesionales de diversas disciplinas en salud. Se incluyeron estudios que pertenecieran a los siguientes niveles de investigación: II (investigaciones experimentales aleatorizadas) y III (investigaciones cuasiexperimentales, como estudios controlados de un solo grupo y series de tiempo)(20). La población de estudio seleccionada en los artículos fueron personas en tratamiento por consumo de alcohol o drogas ilícitas.

Para recabar los artículos de interés se realizó una búsqueda exhaustiva en múltiples bases de datos tales como BioOne, CONRICyT, EBSCO, Elsevier, PubMed, Scielo, Science Direct y Scopus, es importante mencionar que se utilizó el buscador Google Académico como estrategia para la obtener algunos documentos a texto completo. Como estrategia de búsqueda se incluyeron los descriptores DeCS y Mesh en los idiomas inglés (Mindfulness, emotional intelligence, relapse prevention) y español (atención plena, inteligencia emocional, prevención de la recaída) en todas las combinaciones posibles, así mismo se utilizaron truncadores y operadores boléanos en el título y el resumen.

Se obtuvo un total de 471 artículos de todas las bases de datos utilizadas, se procedió a la lectura de títulos, se eliminaron los que no eran de interés y posterior a ello se realizó la lectura de los resúmenes como primer filtro para la selección de los artículos. Posteriormente, se realizó el análisis crítico, la búsqueda de las principales variables y la relación entre el Mindfulness y la Inteligencia Emocional para la prevención de la recaída de consumo de alcohol y drogas ilícitas; se utilizaron herramientas de trabajo como la lectura crítica, subrayado y cuadro de análisis (nivel de evidencia, diseño, tamaño de muestra, instrumentos utilizados y principales resultados encontrados). En la Figura 1 se muestra el proceso de identificación, cribado y elegibilidad, en dónde se puede observar que después de dicho proceso sólo se incluyeron cuatro estudios.

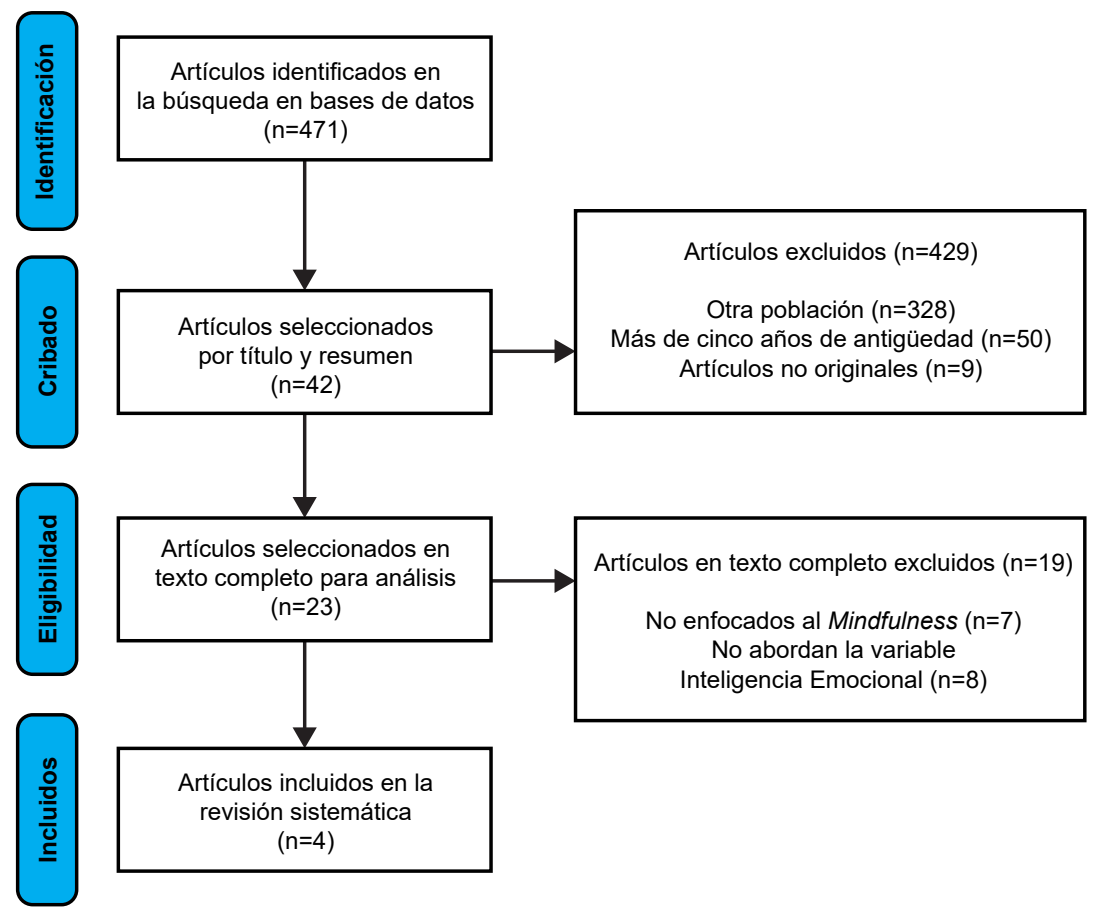

Figura 1 - Proceso de inclusión y exclusión de artículos 


\section{Resultados}

En las Figuras 2 a 4 se presentan las características de los estudios. Se destaca que los estudios fueron realizados en China, India e Irán, con muestras entre los 30 y 89 sujetos, la totalidad de los estudios fueron realizados en hombres que recibían tratamiento forzado o de tipo ambulatorio.

Se encontró que sólo un estudio abordó la prevención de la recaída en el consumo de alcohol(7) y tres estudios en el consumo de marihuana(5-6,8), por cual la mayoría de los estudios recibían tratamiento forzado ${ }^{(5-6,8)}$. En la totalidad de los estudios se mostraron resultados significativos en la regulación de las emociones ${ }^{(5-8)}$, sin embargo, sólo tres estudios mostraron resultados sobre la prevención de la recaída ${ }^{(5-6,8)}$ y sólo un estudio demostró eficacia en el seguimiento postintervención (Figura 5) ${ }^{(5)}$.

Es importante mencionar que sólo tres estudios explicaban de manera detallada la intervención realizada enfocada en Mindfulness ${ }^{(5-6,8)}$. Así también, es importante mencionar que no sólo se realizan actividades de atención plena, sino que es un elemento utilizado dentro de las intervenciones cognitivo conductuales comúnmente realizadas en los pacientes en tratamiento forzado o de tipo ambulatorio(5-8).

\begin{tabular}{|c|c|c|c|c|c|}
\hline Auto, año, lugar & $\begin{array}{l}\text { Tipo de } \\
\text { Droga }\end{array}$ & Diseño y métodos & Indicadores empíricos & Resultados & \begin{tabular}{|c|}
$\begin{array}{c}\text { Seguimiento } \\
\text { Post- } \\
\text { intervención }\end{array}$ \\
\end{tabular} \\
\hline $\begin{array}{l}\text { Chen J, et al. }(2019)^{(5)} \\
\text { China }\end{array}$ & Heroína & $\begin{array}{l}\text { Diseño: Experimental } \\
\text { Muestreo: Aleatorio } \\
\text { simple } \\
\text { Muestra: } 89 \text { hombres } \\
\text { consumidores de } \\
\text { heroína de un centro de } \\
\text { rehabilitación forzado ( } n= \\
46 \text { grupo experimental, } n= \\
43 \text { control). }\end{array}$ & $\begin{array}{l}\text { Escalera de } \\
\text { Contemplación (CL) } \\
\text { Escala de Uso } \\
\text { Obsesivo Compulsivo } \\
\text { de Drogas (OCDUS) } \\
\text { Inventario de Depresión } \\
\text { de Beck (BDI) } \\
\text { Cuestionario de } \\
\text { Agresión (AQ) }\end{array}$ & $\begin{array}{l}\text { Diferencias significativas }(p<0.001) \\
\text { en todos los instrumentos de medición } \\
\text { colocados al grupo experimental pre-test. } \\
\text { Así como al comparar los resultados con } \\
\text { el grupo control pre-test. } \\
\text { Los resultados de la Ecuación de } \\
\text { Esitmación Generalizada (GEE) } \\
\text { mostraron que el grupo experimental tuvo } \\
\text { un efecto significativamente mayor sobre } \\
\text { la abstinencia de drogas que el grupo } \\
\text { control }(p=0.027) \text {. }\end{array}$ & $\begin{array}{c}\mathrm{Si} \\
1,3,6,12,24 \\
\text { y } 36 \text { meses }\end{array}$ \\
\hline
\end{tabular}

Figura 2 - Información relevante de los cuatro estudios incluidos en esta revisión sistemática

\begin{tabular}{|c|c|c|c|c|c|}
\hline Auto, año, lugar & $\begin{array}{l}\text { Tipo de } \\
\text { Droga }\end{array}$ & Diseño y métodos & $\begin{array}{l}\text { Indicadores } \\
\text { empíricos }\end{array}$ & Resultados & $\begin{array}{l}\text { Seguimiento } \\
\text { Post- } \\
\text { intervención }\end{array}$ \\
\hline $\begin{array}{l}\text { George B. }(2015)^{(7)} \\
\text { India }\end{array}$ & Alcohol & $\begin{array}{l}\text { Diseño: Experimental } \\
\text { Muestreo: Aleatrorio } \\
\text { Muestra: } 32 \text { hombres } \\
\text { alcohólicos de } 30 \text { a } \\
50 \text { años ( } n=16 \text { grupo } \\
\text { experimental, } n=16 \\
\text { control). }\end{array}$ & $\begin{array}{l}\text { Prueba de } \\
\text { Identificación de } \\
\text { Trastornos por } \\
\text { Consumo de Alcohol } \\
\text { (AUDIT) } \\
\text { Escala de Abstinencia } \\
\text { del Instituto Clínico de } \\
\text { Evaluación (CIWA-Ar) } \\
\text { Escala de Dificultades } \\
\text { en la Regulación de } \\
\text { las Emociones (DERS) }\end{array}$ & $\begin{array}{l}\text { Diferencias significativas ( } t=135.53 \\
p=0.00) \text { entre el grupo experimental } \\
(\mathrm{M}=46.81, D E=2.40) \text { y control } \\
(M=159.00, D E=2.28) \text { en el puntaje } \\
\text { total del DERS y en sus subescalas. } \\
\text { Diferencias significativas }(t=141.69 \\
p=0.00) \text { en el pre-test }(M=160.31 \\
D E=3.04) \text { y post-test }(M=46.81 \\
D E=2.40) \text { del grupo experimental en el } \\
\text { puntaje total del } D E R S \text { y en sus subescalas. } \\
\text { La efectividad del tratamiento mostró valores } \\
\text { en la prueba de Cohen por encima de } 0.8, \text { lo } \\
\text { cual es considerado como un efecto alto. }\end{array}$ & No \\
\hline
\end{tabular}

Figura 3 - Información relevante de los cuatro estudios incluidos en esta revisión sistemática (continuación)

\begin{tabular}{|c|c|c|c|c|c|}
\hline Auto, año, lugar & $\begin{array}{c}\text { Tipo de } \\
\text { Droga }\end{array}$ & Diseño y métodos & $\begin{array}{l}\text { Indicadores } \\
\text { empíricos }\end{array}$ & Resultados & $\begin{array}{c}\text { Seguimiento } \\
\text { Post- } \\
\text { intervención }\end{array}$ \\
\hline $\begin{array}{l}\text { Esmaeili A, } \\
\text { Khodadadi M, Norozi } \\
\text { E, Reza M. }(2017)^{(6)} \\
\text { Irán }\end{array}$ & Heroína & $\begin{array}{l}\text { Diseño: Cuasiexperimental } \\
\text { Muestreo: No probabilistico } \\
\text { Muestra: } 60 \text { hombres de } \\
20 \text { a } 50 \text { años de un centro } \\
\text { de adicciones ( } n=30 \\
\text { grupo experimental, } n=30 \\
\text { control). }\end{array}$ & $\begin{array}{l}\text { Cuestionario Cognitivo } \\
\text { de Regulación } \\
\text { Emocional [CERO, } \\
\text { (Garnefski \& Kraaij, } \\
\text { 2007)] }\end{array}$ & $\begin{array}{l}\text { Diferencia significativa }(p<0.01) \text { y con } \\
\text { la corrección de Bonferroni }(p<0.006) \\
\text { entre el grupo exprimental y control, en } \\
\text { los puntajes pre-test y post-test de la } \\
\text { regulación positiva ( } 48.2 \% \text { de la varianza } \\
\text { explicada) y negativa }(64.7 \% \text { de la } \\
\text { varianza explicada). }\end{array}$ & No \\
\hline $\begin{array}{l}\text { Zargar F, Bagheri N, } \\
\text { Javad TM, Salehi M. } \\
(2019)^{(8)} \\
\text { Irán }\end{array}$ & Heroína & $\begin{array}{l}\text { Diseño: Experimental } \\
\text { Muestreo: Aleatorio } \\
\text { Muestra: } 30 \text { hombres de } \\
20 \text { a } 50 \text { años de un centro } \\
\text { de adicciones ( } n=15 \\
\text { grupo experimental, } n=15 \\
\text { control). }\end{array}$ & $\begin{array}{l}\text { Escala de Dificultades } \\
\text { en la Regulación de } \\
\text { las Emociones (DERS) } \\
\text { Escala de Ajuste } \\
\text { Diádico (DAS) } \\
\text { Cuestionario de } \\
\text { Creencias sobre } \\
\text { Craving (CBQ) }\end{array}$ & $\begin{array}{l}\text { Diferencias significativas } \\
(F=21.88, p=0.001) \text { en el pre-test } \\
(M=116.6, D E=16.4) \text { y post-test } \\
(M=56.66, D E=18.39) \text { del grupo } \\
\text { experimental en el puntaje total del } C B Q . \\
\text { Mientras que el grupo control no mostró } \\
\text { diferencias significativas en el pre-test } \\
(M=119.1, D E=11.64) \text { y post-test } \\
(M=105.2, D E=34.5) .\end{array}$ & No \\
\hline
\end{tabular}

Figura 4 - Información relevante de los cuatro estudios incluidos en esta revisión sistemática 


\begin{tabular}{|l|c|c|}
\hline \multirow{2}{*}{ Autor } & \multicolumn{2}{c|}{ Mindfulness } \\
\cline { 2 - 3 } & IE & $\mathbf{R}^{\ddagger}$ \\
\hline Chen et al. (2019) & ${ }^{*}$ & ${ }^{*}$ \\
\hline George (2015) & ${ }^{*}$ & ${ }^{+}$ \\
\hline Esmaeili, Khodadadi, Norozi y Raza (2017) & ${ }^{*}$ & ${ }^{*}$ \\
\hline Zargar, Bagheri, Javad y Mehrdad (2019) & ${ }^{*}$ & ${ }^{*}$ \\
\hline
\end{tabular}

Figura 5 - Efecto del Mindfulness sobre la inteligencia emocional y la recaída

\section{Discusión}

La presente revisión otorga evidencia de que la práctica de Mindfulness ha sido utilizada en el tratamiento de la persona con adiciones, reforzando la Inteligencia Emocional y ayudando en la prevención de recaídas(5-8); lo que resulta importante mencionar, ya que la práctica de Mindfulness aunada al incremento de las habilidades de la $\mathrm{IE}$, como la descodificación, reconocimiento y manejo de las emociones protagonizan un papel clave en el tratamiento del trastorno por uso de sustancias(5-12).

Otro aspecto a considerar en la revisión es el enfoque específico por sustancias en el tratamiento del alcohol(7) y heroína ${ }^{(5-6,8)}$ que de acuerdo a los contextos en donde fueron realizadas son las drogas que mayormente se consumen, así como las que presentan mayor dependencia y requieren tratamiento; sin embargo sería interesante conocer los efectos del Mindfulness y la IE en personas que consumen marihuana, al ser una de las drogas de mayor consumo en México(3). En relación con las muestras, se observó que en su totalidad eran hombres( ${ }^{(5-8)}$, lo cual es congruente con la literatura al ser quienes más solicitan tratamiento por dependencia a las drogas.

Los resultados muestran que el Mindfulness muestra un efecto sobre la regulación de las emociones, es decir aumenta la IE de las personas que requieren tratamiento, y su vez la IE media el efecto sobre la prevención de las recaídas ${ }^{(5-8)}$ por lo cual es importante que la práctica del Mindfulness sea incorporada en las intervenciones que se realizan comúnmente con este tipo de población, sin embargo no reemplaza dicha intervención, sino se demuestra que es un componente importante para el tratamiento en personas con dependencia al alcohol y drogas que se debe de continuar investigando con la finalidad de mostrar la eficacia en diversos contextos.

Otro de los hallazgos es que las intervenciones son realizadas en su mayoría por profesionales de psicología(5-8). En este sentido, es importante que los profesionales de enfermería al ser quienes se encargan de atender a las personas de forma global, este tipo de intervenciones se conviertan en un campo importante de actuación, ya que podría incorporarse a este tipo de intervenciones y otorgar el cuidado de manera holística.

\section{Conclusión}

De acuerdo con el análisis de los estudios se puede concluir que la práctica de Mindfulness muestra resultados significativos en la regulación de emociones lo cual aumenta la Inteligencia Emocional de las personas que lo practican y a su vez podría resultar como una herramienta para la prevención de la recaída en personas que se encuentran en tratamiento por consumo de alcohol o drogas.

La evidencia existente menciona que los hombres son quienes más solicitan tratamiento, lo cual se observó en los estudios al encontrar que en la totalidad de los estudios las muestran eran hombres adultos. Por lo cual se sugiere que se realicen investigaciones en mujeres con la finalidad de conocer la eficacia en esta población.

No se encontraron estudios realizados en contextos mexicanos o en Latinoamérica, por lo que resulta interesante replicar dichas intervenciones con la finalidad de seguir realizando investigaciones enfocadas en la práctica de Mindfulness y que ayuden en el aumento de la IE, ya que se considera como una buena herramienta que muestra resultados prometedores en la prevención de la recaída. Sin embargo, es necesario consolidar los resultados, por lo cual se requiere y se sugiere la evaluación de un número mayor de estudios empíricos, en diferentes contextos.

\section{Referencias}

1. Organización Mundial de la Salud. Alcohol [internet]; 2018 [cited 28 febrero 2020]. Disponible en: https:// www.who.int/es/news-room/fact-sheets/detail/alcohol

2. Oficina de las Naciones Unidas contra la Droga y el Delito. Informe Mundial sobre las Drogas [internet]; 2017 [cited 27 febrero 2020]. Disponible en: https:// www.unodc.org/wdr2017/field/WDR_Booklet1_Exsum_ Spanish.pdf

3. Instituto Nacional de Psiquiatría Ramón de la Fuente Muñiz; Instituto Nacional de Salud Pública, Comisión Nacional Contra las Adicciones, Secretaría de Salud. Encuesta Nacional de Consumo de Drogas, Alcohol y Tabaco 2016-2017: Reporte de Drogas. VillatoroVelázquez JA., Resendiz-Escobar, E., Mujica-Salazar, A., Bretón-Cirett, M., Cañas-Martínez, V., Soto-Hernández, I., Fregoso-Ito, D., Fleiz-Bautista, C., Medina-Mora ME., Gutiérrez-Reyes, J., Franco-Núñez, A., Romero-Martínez, M. \& Mendoza-Alvarado, L. Ciudad de México, México: INPRFM; 2017. Disponible en: www.inprf.gob.mx, www. conadic.gob.mx, www.insp.mx

4. National Institute of Drug Abuse. The Science of drug y abuse. Alcohol [internet]; 2011 [cited 02 Febrero 2020]. Disponible en: http://www.drugabuse.gov/ drugs- abuse/alcohol

5. Chen J, Yu J, Ciao J, Xiao Y, Gu H, Zhong R, et al. Abstinence following a motivation-skill-desensitizationmental energy intervention for heroin dependence: A 
three year follow-up result of a randomized controlled trial. Current Medical Science. 2019; 39(3): 472-482. doi: 10.1007/s11596-019-2062-y.

6. Esmaeili A, Khodadadi M, Norozi E, Reza MM. Effectiveness of Mindfulness based cognitive group therapy on cognitive emotion regulation of patients under treatment with methadone. Journal of Substance Use. 2017; 03(49): 1-5. doi: 10.1080/14659891.2017.1348553

7. George B. Efficacy of acceptance and Mindful based relapse prevention program on emotion regulation difficulty among alcoholics in Kerala India. J Alcohol Drug Depend. 2015; 3(3): 2-11. doi: 10.4172/2329-6488.1000205

8. Zargar F, Bagheri N, Javad TM, Salehi M. Effectiveness of emotion regulation group therapy on craving, emotion problems, and marital satisfaction in patients with substance use disorders: A randomized clinical trial. Iran J Psychiatry. [Internet] 2019; [cited 28 febrero 2020]; 14(4): 283-290. Disponible en: https://www.ncbi.nlm. nih.gov/pmc/articles/PMC7007510/

9. Witkiewitz K, Stein ER, Votaw VR, Wilson AD, Ross $C R$, Gallegos SJ, et al. Mindfulness-based relapse prevention and transcranial direct current stimulation to reduce heavy drinking: A double-blind sham-controlled randomized trial. Alcohol Clin Exp Res. 2019; 43(6): 1296-1307. doi: 10.1111/acer.14053

10. Grant S, Colaiaco B, Motala A, Shariman R, Booth M, Sorbero $M$, et al. Mindfulness-based relapse prevention for substance use disorders: A systematic review and meta-analysis. J Addict Med. 2017; 11(5): 386-396. doi: 10.1097/ADM.0000000000000338.

11. Tang YY, Tang R, Posner MI. Mindfulness meditation improves emotion regulation and reduces drug abuse. Drug and Alcohol Dependence. 2016; 163: S13-S18. doi: 10.1016/j.drugalcdep.2015.11.041.

12. Guendelman S, Medeiros S, Rampes H. Mindfulness and emotion regulation: Insights from neurobiological, psychological, and clinical studies. Front Psychol; 8(220): 1-23. doi: 10.3389/fpsyg.2017.00220

13. Darwin J. Emotional intelligence and Mindfulness [internet]. Sheffield (UK). Centre for Mindful life enhancement; 2015 [cited 28 febrero 2020]. Disponible en: https://pdfs.semanticscholar.org/1130/06167c7d3f5 d2480458e1eb882fe197d6a7b.pdf

14. Enriquez $H$, Ramos N, Esparza O. Impact of the Mindful emotional intelligence program on emotional regulation in college students. International Journal of Psychology and Psychological Therapy. [Internet] 2017; [cited 28 febrero 2020]; 17(1): 39-48. Disponible en: https://www.ijpsy.com/volumen17/num1/455.html

15. Bar-On R. BarOn Emotional Quotient Inventory (EQ-i): Technical manual; 1997. Toronto, Canada: Multi-Health Systems. Disponible en: http://www. eiconsortium.org/pdf/baron_model_of_emotional_ social_intelligence.pdf

16. Moscoso MS. Hacía una integración de Mindfulness e inteligencia emocional en psicología y educación. Liberabit. 2019; 25(1): 107-117. doi: 10.24265/ liberabit.2019.v25n1.09

17. Ramos DN, Salcido CL. Programa Inteligencia Emocional Plena (PINEP) aplicando Mindfulness para regular emociones. Revista de Psicoterapia. 2017; 28(107): 259-270. doi: https://doi.org/10.33898/rdp. v28i107.152

18. Higgins J, Thomas J. Cochrane handbook for systematic reviews of interventions. 2019 [citado 28 febrero 2020]. Disponible en: https://training.cochrane. org/handbook/current

19. Armendáriz GNA, Rodríguez PLA, Guzmán FFR, López CMA, Ahumada CJG, Alonso CMA. Revisiones sistemáticas en el fenómeno de drogas. En experiencias de investigación de la red mexicna de facultades y escuelas de enfermeríua: predicción del consumo de alcohol y tabaco en jóvenes de preparatoria. Monterrey (MX): Editorial Universitaria; 2015. p. 31-41.

20. Stetler ChB, Bruneell M, Giuliano K, Newell V. Evidence-based practice and the role of nursing leadership. The Journal of Nursing Administration. 1998; 28(8), 45-53. doi: 10.1097/00005110-199807000-00011

\section{Contribución de los Autores}

Concepción y dibujo de la pesquisa: Julia Lizeth Villarrea Mata y Edna Idalia Paulina Navarro Oliva. Redacción del manuscrito: Julia Lizeth Villarrea Mata y Edna Idalia Paulina Navarro Oliva. Revisión crítica del manuscrito en cuanto al contenido intelectual importante: Edgar Bresó Esteve, Linda Azucena Rodríguez Puente, María Magdalena Alonso-Castillo y Reyna Torres Obregón.

Todos los autores aprobaron la versión final del texto.

Conflicto de intereses: los autores han declarado que no existe ningún conflicto de intereses.

Recebido: 03.03.2020

Aceito: 06.07.2020 